\title{
Modeling association between times to recurrence of the different polarities in bipolar disorder among service seekers in urban Nigeria: a survival analysis approach
}

\author{
This article was published in the following Dove Press journal: \\ Neuropsychiatric Disease and Treatment \\ 21 July 2017 \\ Number of times this article has been viewed
}

\author{
Adeniyi Francis \\ Fagbamigbe' \\ Victor Adesola Makanjuola ${ }^{2}$ \\ 'Department of Epidemiology \\ and Medical Statistics, Faculty of \\ Public Health, College of Medicine, \\ University of Ibadan, Nigeria; \\ ${ }^{2}$ Department of Psychiatry, College \\ of Medicine, University of Ibadan, \\ Ibadan Nigeria
}

Introduction: Bipolar disorder (BD) remains both a clinical and public health challenge worldwide, especially in developing countries such as Nigeria. Many studies have focused on prevalence and recurrences among BD service seekers but little has been documented on the nature, strength, direction, existence, and estimation of association between times to recurrence of the two possible polarities or mood episodes in BD. In this study, we explored the association between durations before recurrence of depression and manic episodes among people seeking treatment for BD.

Methods: This analytical study used retrospective data of 467 persons who sought treatment for BD at the psychiatric clinic of University College Hospital, Ibadan, Nigeria between 2005 and 2014. Descriptive statistics were used to explore the data. We right-censored the data and obtained Kaplan-Meier estimates of the time to recurrence of the outcomes and transformed the estimates to standardized binormal data using quantile-quantile transformation. The likelihood was maximized to obtain the maximum likelihood estimate of the association parameter at $5 \%$ significance level.

Results: The mean ( \pm standard deviation) age of the respondents was $32.9 \pm 12.9$ years, this was lower among service seekers who were initially diagnosed with mania than among those initially diagnosed with depression $(31.3 \pm 11.6,33.2 \pm 11.9$, respectively). The median survival time to recurrence of mania and depression among the patients was 1,120 and 745 days, respectively, whereas association between times to recurrence of mania and depression was maximized at 0.67 (95\% confidence interval: $0.62-0.71$ ).

Conclusion: There exists a strong and positive association between times to recurrence of depression and mania in $\mathrm{BD}$. The longer the time to recurrence of mania, the longer the time to recurrence of depression and vice versa.

Keywords: bipolar disorder, time to recurrence, depression, bivariate censored outcomes, maximum likelihood estimates, mania

\section{Introduction}

Bipolar disorder (BD) is a psychiatric condition affecting mood which can be defined by the presence of one or more episodes of abnormally elevated energy levels (manic), cognition, and mood with or without one or more depressive episodes. ${ }^{1-6}$ In the manic phase, a person might feel ecstatic, self-important, and energetic; but when the person becomes depressed, the mood shifts to extreme sadness, negative thinking, and indifference to things that used to bring them happiness. ${ }^{7}$ A recent systematic review of
Fagbamigbe

Department of Epidemiology and Medical Statistics, Faculty of Public

Health, College of Medicine, University

of Ibadan, Ibadan, Nigeria

Tel +2348061348I65

Email franstel74@yahoo.com 
prevalence and burden of BD in Africa showed that lifetime prevalence of BD was $0.1 \%-1.83 \%{ }^{6}$ Mania is much less common than depression. The recurrence rates for mania and depression in BD are high and were put at around 50\% at 1 year and $70 \%$ at 4 years. ${ }^{2,8}$ The lifetime prevalence of BD type-1, which includes at least one life-time manic episode, has been estimated at $1 \% .{ }^{9}$ A re-analysis of data from the National Epidemiological Catchment Area survey in the United States, however, suggested that $0.8 \%$ experience a manic episode at least once (the diagnostic threshold for BD type-1) and $0.5 \%$ for hypomanic episode (the diagnostic threshold for BD type-2 or cyclothymia). ${ }^{10}$

Recurrence is the primary issue in BD. After a successive treatment, a patient with BD may have a recurrence of either mania, depression, or both (mixed episode). Pharmacotherapy with mood stabilizers such as carbamazepine and antipsychotics such as risperidone is the mainstay of treatment in the facility. The recurrence outcomes in a particular patient with BD monitored overtime would naturally fall into one of four possible scenarios. ${ }^{2,3}$ The patient could either develop mania alone or depression alone, both (bad outcome), or no recurrence at all (good outcome). Risk factors for the recurrence of BD have been identified to include arbitrary discontinuation or lowering one's dose of medication, under or over medication, inconsistent sleep schedule, inadequate stress management, and poor lifestyle choices. ${ }^{4,11,12}$ Also considered in the study of BD was the duration or period of when the last episode of depression or mania occurred.

Several studies have consistently reported that different socio-economic problems influence recurrences and polarity of episodes among people being treated for BD. ${ }^{3,6,11,12}$ A recent report on trial performed to equip community mental health teams on prevention of enhanced recurrence had indicated that adequate prevention of recurrences of mood episodes in BD could increase time to recurrence. ${ }^{2}$ However, there remained unanswered questions about timing of recurrence in BD. For instance, what type of relationship exists between the polarities of mood episodes when recurred? What are the relationships between recurrences of mood episodes? What is the duration before recurrence? What is the strength of association between the duration before recurrence of the different polarities in $\mathrm{BD}$ as well as what contributes to the timing of these recurrences?

Hitherto, majority of the earlier studies have focused primarily on prevalence of recurrences in BD while very little work has been done in studying dependence structures between time to recurrence of the two possible poles of mood in BD especially in Nigeria and Africa. The study of the dependence structure of time to recurrence of mania and depression provides an insight regarding the dependence of recurrence of one of the outcomes on the other, as well as provides a clue on causes and timings of the recurrences. This might aid in the management of persons with $\mathrm{BD}$ and help to reduce the degree as well as the frequency of the recurrences. We hypothesized that there is no association between time to recurrence of depression and mania in BD. In this study, we aimed to obtain median survival time to recurrence of episodes in BD and to determine the existence, direction, as well as strength of association between censored time to recurrence in such episodes.

\section{Methods}

Retrospective data were extracted from records of patients who were diagnosed, treated, and managed for BD in the Psychiatric Clinic of University College Hospital, Ibadan between 2005 and 2014. The diagnosis were made by practicing senior psychiatrists after detailed clinical interview and examination of the mental state followed by physical examination based on the criteria set by the fourth version of the Diagnostic and Statistical Manual of Mental Disorders criteria (DSM IV) and the 10th version of the International Classification of Diseases (ICD 10). To ensure a possibility of recurrence, we selected the records of only the patients who had earlier experienced at least one episode of either mania or depression and were successfully managed. In all, we collected data from records of 467 patients. We recorded patients' time to recurrence (days) into either of the moods as well as other characteristics. Patients with comorbidities such as alcohol or drug abuse/dependence were excluded to minimize the confounding effect of comorbidities.

A patient with BD followed over time may or may not experience either of the mood disorders. In a situation where there is no recurrence into either or both of the outcomes, the time to recurrence is censored and incorporated into the analysis. In survival analysis, observations were censored when only partial information on survival times was available. This often arises when 1) study ends, and the event has not been experienced; 2) subject withdraws from the study and 3) subjects are lost to follow-up. Like failure time, censoring is a point event and the period of observation for censored individuals must also be recorded and analyzed along with others with complete information.

\section{Statistical analysis}

There are four possible outcomes in a bivariate survival analysis. We denoted the censoring index of the four distinct 
outcomes as following: 11 -when both mania and depression were observed, 10 - mania was observed and depression was censored, 01 - mania was censored and depression was observed, and 00 - when both mania and depression were censored. ${ }^{13,14}$ We defined time to an event as the number of days between baseline (time of a successful treatment) to the time of first recurrence. The participants who had no recurrence were censored and their survival time is the duration between time of a successful treatment and the date "last seen." In analysis of depressive recurrence, a case with any manic, hypomanic, or mixed episode occurring before the depressive recurrence are treated as censored at the time of nondepressive recurrence and vice versa. ${ }^{15}$ In this study, we focused on the first recurrence. We did not include instances of multiple recurrences in our analysis so as to avoid unnecessary inflation of intra-correlation, which might be caused due to the repeated measurements from same individuals.

We used the model developed and experimented in Fagbamigbe and Adebowale for our analysis. ${ }^{14}$ The model relied largely on the principle of optimization using a maximum likelihood estimator. The maximum likelihood estimator searches between a predefined minimum and maximum value for the estimate and converges on the value that has maximum likelihood for the estimate. In this study, we set minimum value as -1.00 and maximum value as +1.00 , that is, the only possible range for correlation coefficient. We obtained Kaplan-Meier estimates of both mania and depression and transformed the estimates to binormal data since Kaplan-Meier estimates are exponential in distribution using quantile-quantile (inversion of Kaplan-Meier estimates) transformation. R statistical software was used to perform Kaplan-Meier estimation, normalization and optimization.

Let mania be $x$ and depression be $y$. Furthermore, let us denote the likelihoods of the four scenarios narrated above with $H_{1}(x, y), H_{2}(x, y), H_{3}(x, y)$ and $H_{4}(x, y)$ such that

$$
\begin{gathered}
H_{1}(x, y)=f(x, y ; \rho)=\frac{1}{2 \pi \sqrt{1-\rho^{2}}} \exp \left(-\frac{x^{2}+y^{2}-2 \rho x y}{2\left(1-\rho^{2}\right)}\right) \\
H_{2}(x, y)=\int_{c_{2}}^{\infty} f(x, y ; \rho) d y=f(x)\left[1-F_{Y \mid X=x}\left(c_{2}\right)\right] \\
H_{3}(x, y)=\int_{c_{1}}^{\infty} f(x, y ; \rho) d x=f(y)\left[1-F_{X \mid Y=y}\left(c_{1}\right)\right] \\
H_{4}(x, y)=\int_{c_{2}}^{\infty} \int_{c_{1}}^{\infty} f(x, y ; \rho) d x d y \\
=1-F_{X}\left(c_{1}\right)-F_{Y}\left(c_{2}\right)+F_{X Y}\left(c_{1}, c_{2} ; \rho\right)
\end{gathered}
$$

Neuropsychiatric Disease and Treatment 2017:13

submit your manuscript | www.dovepress.com where,

$C_{1}=$ the censoring time for mania $(\mathrm{X})$

$C_{2}=$ the censoring time for depression (Y)

$F_{x}\left(c_{1}\right)$ is the normal cumulative distribution function of mania at $c_{1}$

$F_{y}\left(c_{2}\right)$ is the normal cumulative distribution function of depression at $c_{2}$

$F_{X \mid Y=y}\left(c_{1}\right)$ is the marginal distribution function of mania at $c_{1}$ given depression

$F_{Y \mid X=x}\left(c_{2}\right)$ is the marginal distribution function of depression at $c_{2}$ given mania

$F_{X, Y}\left(c_{1}, c_{2} ; \rho\right)$ is the bivariate cumulative distribution function of mania and depression at $c_{1}$ and $c_{2}$ respectively with correlation $\rho$.

and

$$
\begin{aligned}
f_{X, Y}(x, y)= & \frac{1}{2 \pi \sqrt{1-\rho^{2}}} \exp \left(\frac { - 1 } { 2 ( 1 - \rho ^ { 2 } ) } \left[\left(\frac{x-\mu_{x}}{\sigma_{x}}\right)^{2}\right.\right. \\
& \left.\left.+\left(\frac{y-\mu_{y}}{\sigma_{y}}\right)^{2}-2 \rho\left(\frac{x-\mu_{x}}{\sigma_{x}}\right)\left(\frac{y-\mu_{y}}{\sigma_{y}}\right)\right]\right)
\end{aligned}
$$

Then used the multivariate package in R-statistical software to maximize the resulting likelihood function

$$
\mathrm{L}_{j}=H_{1}^{\delta_{1} \delta_{2}} H_{2}^{\delta_{1}\left(1-\delta_{2}\right)} H_{3}^{\left(1-\delta_{1}\right) \delta_{2}} H_{4}^{\left(1-\delta_{1}\right)\left(1-\delta_{2}\right)}
$$

where,

$$
\delta_{i}=\left\{\begin{array}{l}
1 \text { if event of interest was observed } \\
0 \text { if event of interest was censored }
\end{array} \text { for } i=1,2, \ldots \ldots \ldots \ldots \mathrm{n}\right.
$$

For further numerical details, please see Fagbamigbe and Adebowale. ${ }^{14}$

SPSS version 22.0 was used to produce the descriptive statistics while life table module in Stata version 14 was used to compute the median time to recurrence of mania and depression. All statistical significances were determined at $5 \%$

\section{Ethical approval}

Ethical approval to conduct this study was obtained from the Ibadan Joint Ethical Review Board, University College Hospital, University of Ibadan, Nigeria. The need for consent from patients to review their medical records was waived because 1) personal details of the patients were not required and 2) the study took place after the patients were treated and therefore, it was no longer possible to seek their consent. 
To maintain confidentiality, the names of patients were not retrieved. The retrieved data were kept confidential and only accessible to research team members. The data in the extraction form were entered into a password-protected computer that was accessible to the researchers only.

\section{Results}

A total of 467 patients with BD met the inclusion criteria and were therefore analyzed in this study. These patients had suffered at least one episode of either mania or depression in BD. Of the 467 service seekers, 183 (39.0\%) were initially diagnosed with mania, whereas the remainder of 284 (60.8\%) were initially diagnosed with depression. The overall mean $( \pm$ standard deviation $[S D])$ age of the patients was $32.92( \pm 12.89)$ years, whereas mean age for patients with an index case of mania and depression was $30.46( \pm 10.75)$ and $34.50( \pm 13.87)$ years, respectively. The differences between these ages were statistically significant $(P<0.001)$. Service seekers initially diagnosed with mania were found to be younger than those who had depression. Over half of those who were diagnosed with mania were younger than 30 years whereas over half of those with depression were aged 30 years or older. About $65.0 \%$ of those initially diagnosed with mania were singles, whereas $47.5 \%$ among those with depression were married (data not shown in tables).

Of the 467 service seekers who were followed up, there were $173(37.0 \%)$ patients who did not recur into either mania or depression during the follow-up. However, 52 (11.1\%) of them suffered both mania and depression during their follow-up (Table 1). The association between recurrence of mania and depression was statistically significant $(P<0.05)$. However, a McNemar test performed to determine if relationship exist between recurrence types and whether the recurrence was actually observed was not statistically significant as $\left(\chi^{2}=3.012\right)$ with one degree of freedom, two-tailed $P>0.05$ and odds ratio of 1.262 (95\% confidence interval [CI]: 0.972-1.642).

Table I Chances of recurrence of mania and depression among the bipolar disorder service seekers

\begin{tabular}{llll}
\hline Outcomes & \multicolumn{2}{l}{ Depression } \\
\cline { 2 - 4 } & No recurrence & Recurrence & Total \\
\hline Mania, n (\%) & & & \\
No recurrence & $173(37.1)^{*}$ & $135(28.9)^{*}$ & $308(66.0)$ \\
Recurrence & $107(22.9)^{*}$ & $52(1 \mathrm{I} .1)^{*}$ & $159(34.0)$ \\
Total, N (\%) & $280(60.0)$ & $187(40.0)$ & 467 \\
\hline
\end{tabular}

Note: *Overall percentages.
Considering the patients who were diagnosed with mania at the time of presentation, the probability of recurrence of mania during follow-up was found to be 0.404 . In addition, the chances of patients who had earlier experienced mania to recur into depression was found to be 0.421 . The remaining patients constituting 0.175 did not experience any of the mood episode in BD. The probability of a patient who had earlier experienced depression suffering another episode of depression during the follow-up was 0.387 . This is higher than the probability of a patient who had earlier experienced depression to recur into mania (0.299). Other patients did not experience any of the polarities in BD again. The McNemar test of association with correction for continuity showed that recurrence of polarities in $\mathrm{BD}$ was statistically significant $(P<0.05)$ among patients with initial diagnosis of depression but not significant among those who were initially diagnosed with mania (data not shown in tables).

Collectively, 415 patients who constituted about 9 in every 10 BD service seekers who were followed up experienced at least one episode of $\mathrm{BD}$ (either depression or manic). The mean $( \pm \mathrm{SD})$ age of patients who recurred into mania alone, depression alone, none, and both was 31.3 \pm 11.6 , $33.2 \pm 11.9,33.3 \pm 13.6$ and $32.42 \pm 9.3$ years, respectively. Using life table techniques, we obtained the median time to recurrence of mania and depression as 1,120 and 745 days, respectively (Table 2 ).

Figure 1 shows the distribution of time to recurrence of mania and depression. Depression recurrence seemed to be shorter than recurrence of mania.

As shown in Figure 2, the survivorship of mania seems longer than survivorship of depression.

\section{Exploring survival functions of the data at $0 \%$ and $50 \%$ censoring}

Figure 3 shows the three survival curves for each of the three different possible censoring indexes. The first curve (in red color) shows the survival function of the original data when there are equal chances of "failure" or censoring the

Table 2 Descriptive statistics of time to recurrence of bipolar disorder

\begin{tabular}{lcc}
\hline Statistics & \multicolumn{2}{c}{ Recurrences } \\
\cline { 2 - 3 } & Mania & Depression \\
\hline Mean & 613.07 & 575.134 \\
Standard error of mean & 32.18 & 31.18 \\
Standard deviation & 695.36 & 673.75 \\
Median & $1,120.00$ & 745.00 \\
Mode & 15.00 & 8.00 \\
Range & $2,644.00$ & $2,606.00$ \\
\hline
\end{tabular}




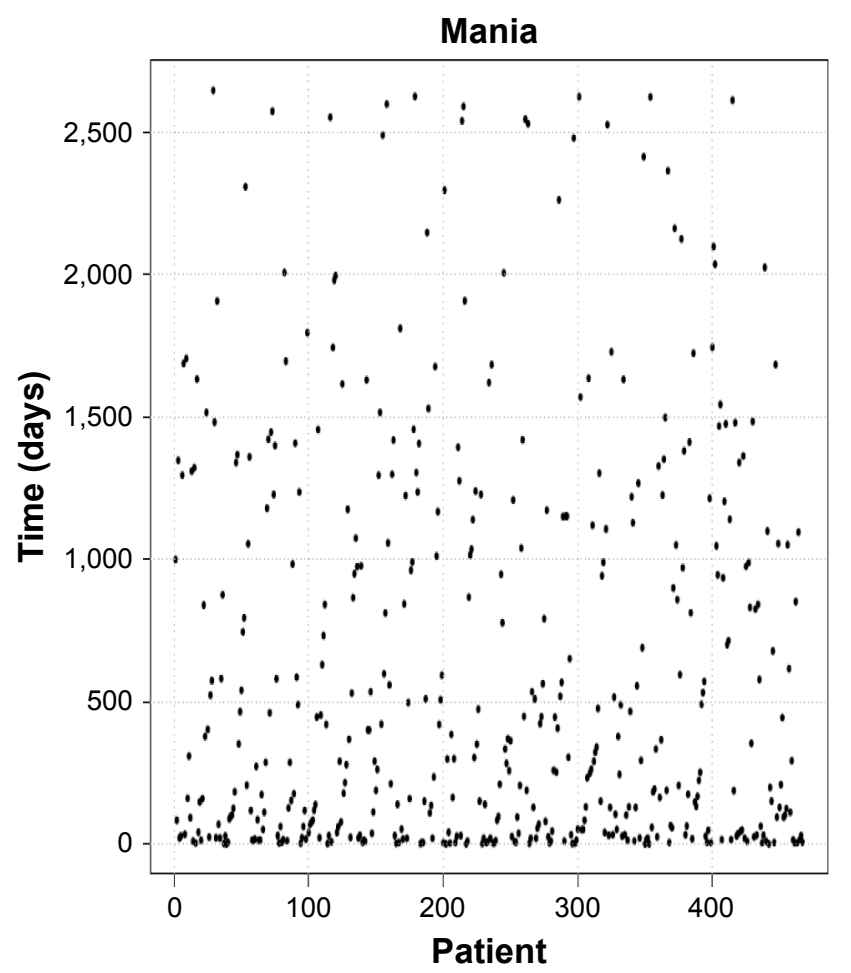

Figure I Failure time of recurrences of bipolar depression among the service seekers.

outcomes of interest. The second curve (in green color) shows a situation when censored observations were ignored, that is, (only the observed events were used while the censored events were ignored), whereas the last curve (in black color) is for situations where all events were censored as failure - a situation that arises when all patients were considered to have all experienced the event of interest.

\section{Maximum likelihood estimation}

Using the procedure described under the statistical analysis methods, the association between time to recurrence in $\mathrm{BD}$

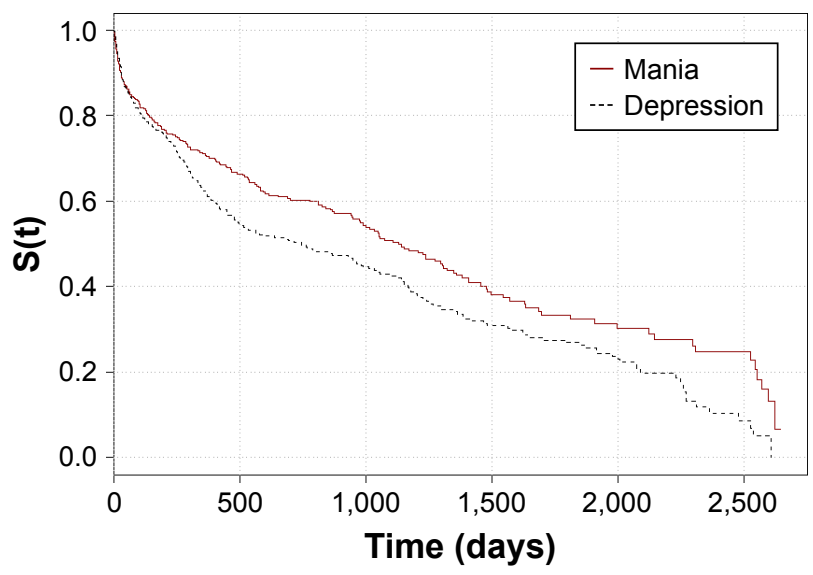

Figure 2 Kaplan-Meier plot of the survival time of recurrences of bipolar disorders. Abbreviation: $\mathrm{S}(\mathrm{t})$, survivorship probability at time $\mathrm{t}$.

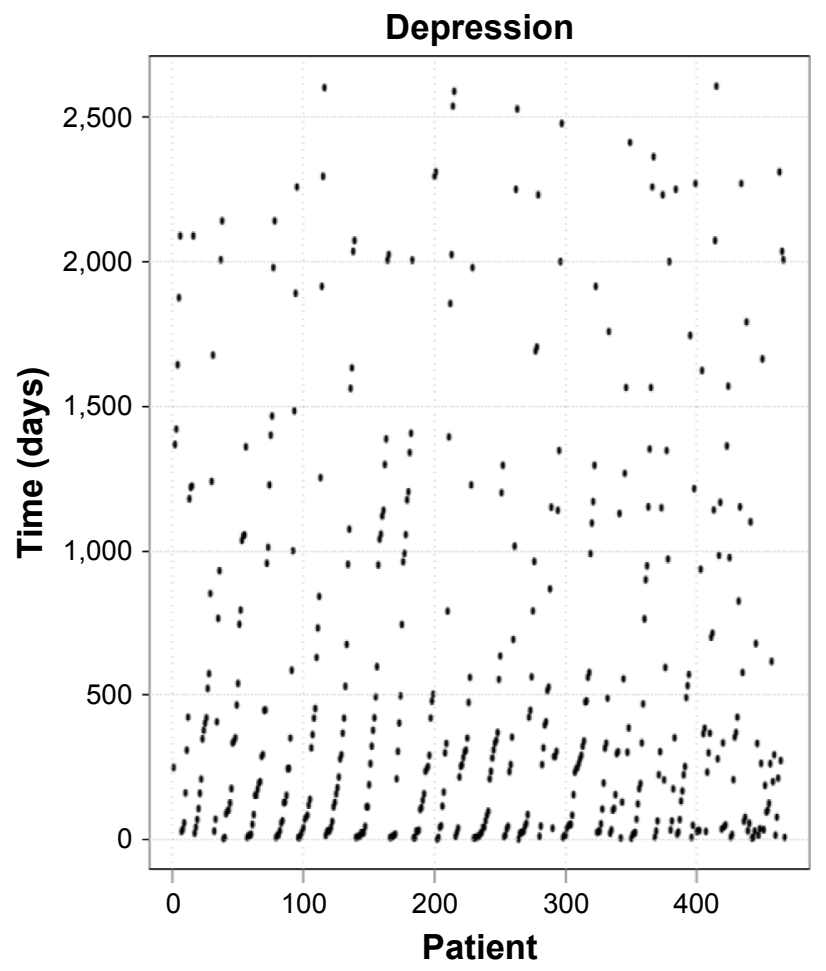

was maximized at 0.6684 with $95 \% \mathrm{CI}=0.6231-0.7137$ (Table 3). In Figure 4, the visual display of the maximum likelihood estimation procedure of the association parameter between the time to recurrence of mania and depression is presented. The likelihoods of the estimate peaked when the association parameter was 0.6684 .

\section{Test of hypothesis of no association}

We tested the null hypothesis $\left(\mathrm{H}_{0}\right)$ that there is no association between the time to recurrence of depression and mania against the alternative hypothesis $\left(\mathrm{H}_{1}\right)$ that association exists between the times to recurrence of different polarities in BD. The test was performed using the estimated association parameter and the number of paired observations $(\mathrm{n}=463)$. We reject the null hypothesis if $P<0.001$ suggesting that there was inherent association between the times to recurrence of different polarities in $\mathrm{BD}$ and that the association was not due to chance.

\section{Discussion}

In this study, we found a significant association between time to recurrence of mania and depression among the service seekers. The association was maximized at 0.6683 , which indicated a positive and strong relationship and that the higher the recurrence time of mania the higher the recurrence time of depression and vice versa. 

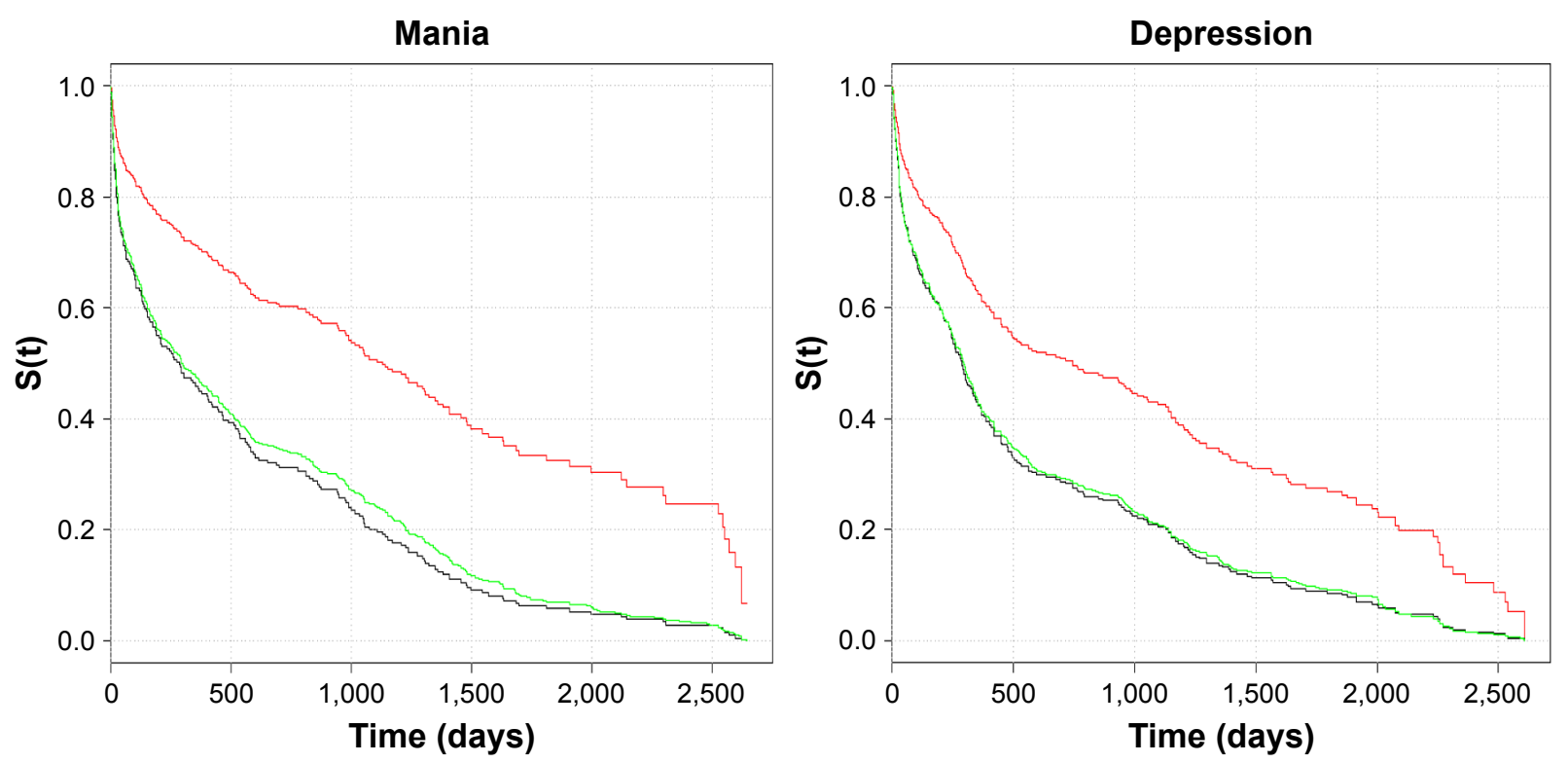

$$
\text { - Original data - Ignoring censoring - Censored as failure }
$$

Figure 3 Kaplan-Meier plot of the survival time of recurrences of bipolar disorders by type. Abbreviation: $\mathrm{S}(\mathrm{t})$, survivorship probability at time $\mathrm{t}$.

Furthermore, the recurrence of depression is much more frequent than the recurrence of mania among patients undergoing treatment for $\mathrm{BD}$. This suggests that a particular $\mathrm{BD}$ service seeker followed up is more likely to experience an episode of depression than experiencing mania. This finding is similar to the findings from an earlier study which reported that recurrence of depression was higher than the recurrence of mania (34.7\% vs $13.8 \%) .{ }^{15}$ Specifically, the chances of relapsing to mania by patients who were initially diagnosed with mania was 0.404 compared with 0.421 for those who were initially diagnosed with depression. But the chance of relapsing to depression by a patient who was initially diagnosed with depression was 0.387 and 0.299 for recurrence of mania. When all service seekers were considered irrespective of their initial diagnosis, the chances for mania and depression were 0.34 and 0.40 , respectively. Recurrence to depression was significantly higher than recurrence to mania $(P<0.05)$. The finding seems to be due to the medical

Table 3 Maximum likelihood estimate of the association between the time to recurrence of depression and mania in bipolar disorder

\begin{tabular}{ll}
\hline Statistics & Estimates \\
\hline Likelihood & $\mathrm{I}, 180.704$ \\
Correlation coeffcient $(\rho)$ & 0.6684 \\
Standard error of $\rho$ & 0.0453 \\
$95 \% \mathrm{Cl}$ for $\rho$ & $(0.623 \mathrm{I}, 0.7 \mathrm{I} 7)$ \\
$P$-value & 0.0000 \\
\hline
\end{tabular}

Abbreviation: $\mathrm{Cl}$, confidence interval. characteristic of depression as it is easier to recur into depression than mania. Our finding is further corroborated by an earlier report that recurrence into depression is more frequent than recurrence into mania. ${ }^{3}$

The finding that about 9 in every 10 BD service seekers followed up experienced at least one episode of recurrence suggested the need for development as well as administration of new interventions to reduce recurrences among the service seekers. The $95 \%$ CI built around the maximized association between the times to recurrence of mania and depression indicated how significant the estimate is. This finding was also supported by the outcome of the test of hypothesis of no association between the times to recurrence which showed that significant association existed between the time to recurrence of mania and depression.

Our finding on existence of positive association between the times to recurrence of mania and depression is similar to previous finding ${ }^{16}$ which utilized methods proposed for measuring correlations in the presence of interval censored data. ${ }^{13,17}$ The method was used to estimate the correlation between ages at occurrence of two distinct observed events, with modifications to accommodate the interval censored nature of the available data. The authors studied characterization of the correlation between ages at entry into breast and pubic hair development. The method worked efficiently, and they ascertained that the likelihood was maximized at correlation $(\rho)=0.503$ to 0.506 . Their findings showed a 


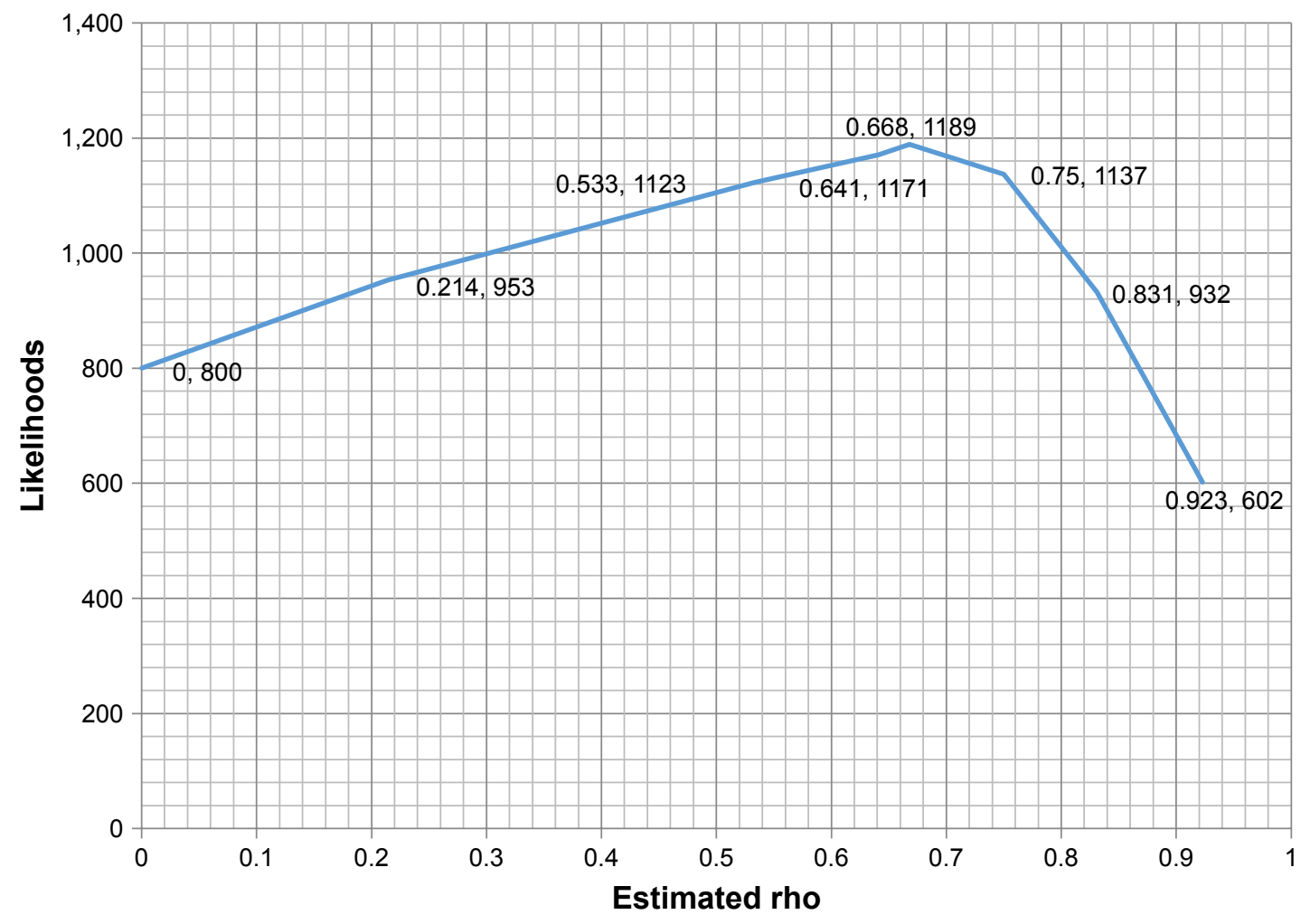

Figure 4 Convergence plot of the maximum likelihood estimate of the association parameter (rho).

positive and strong association between ages at entry into breast and pubic hair development.

This study found a median recurrence time of 1,120 days for mania and 745 days for depression. This implied that higher proportion of manic events reoccur within the first 3 years, which suggests that patients must be monitored more closely during their first 3 years after initial diagnosis and treatment so as to reduce or totally avoid a recurrence of mania. In the same direction, higher proportion of depressive events occurred within the first 2 years, which suggests that patients must be monitored more closely during their first 2 years after initial diagnosis to reduce or totally avoid a recurrence of depression. Our estimated median survival times were similar to the median recurrence time of BD reported earlier. ${ }^{1,18}$ While a previous study affirmed that the median time to recurrence was 87 weeks in a study of 103 individuals with BD type-1 disorder who were psychotic at the time of initial hospitalization and subsequently achieved remission. ${ }^{1}$ Another study reported a mean survival time of about 3 years in an outpatient study of 82 patients that did not examine first-admission patients with BD. ${ }^{18}$

Incomplete data is a major limitation in this study. There was no information in case files of some of the service seekers after the first successful treatment for BD. Despite these limitations, our findings that recurrence of $\mathrm{BD}$ is high with depression being more frequent than mania and that there exist direct and strong association between the time to recurrence of mania and depression are valid as the total number of cases used in this study is large. Our sample size is also relatively large compared with sample sizes used in previous studies.

\section{Conclusion}

There exists a strong association between the time to recurrence of mania and depression among persons living with BD in Nigeria. We opined that the understanding of this association will help the physicians as well as the patients in the moderation and management of recurrence.

\section{Acknowledgments}

The authors acknowledge the support received from staff from the Department of Psychiatry, University College Hospital, Ibadan and the technical support given to the first author by the Consortium for Advanced Research Training in Africa (CARTA). This research was partially funded by a Fellowship award provided by CARTA. CARTA has been funded by the Wellcome Trust (UK) (Grant No: 087547/Z/08/Z), the Department for International Development (DfID) 
under the Development Partnerships in Higher Education (DelPHE), the Carnegie Corporation of New York (Grant No: B 8606), the Ford Foundation (Grant No: 1100-0399), and the Bill and Melinda Gates Foundation (Grant No: 51228).

\section{Disclosure}

The authors report no conflicts of interest in this work.

\section{References}

1. Bromet EJ, Finch SJ, Carlson GA, et al. Time to remission and relapse after the first hospital admission in severe bipolar disorder. Soc Psychiatry Psychiatr Epidemiol. 2005;40(2):106-113.

2. Lobban F, Taylor L, Chandler C, et al. Enhanced relapse prevention for bipolar disorder by community mental health teams: cluster feasibility randomised trial. Br J Psychiatry. 2010;196(1):59-63.

3. Mayo Clinic. Bipolar disorder overview 2017. Available from: www. mayoclinic.org/diseases-conditions/bipolar-disorder/home/ovc20307967. Accessed June 30, 2017.

4. NAMI. (National Alliance on Mental Illness) Understanding Bipolar Disorder and Recovery; 2008. Available from: http://cpidnyc.com/ wp-content/uploads/2013/03/NAMI_Bipolar_Disorder_Aug08.pdf. Accessed June 30, 2017.

5. Studart PM, Filho SB, Studart ABD, Almeida G-DA, MirandaScippa Â. Social support and bipolar disorder. Arch Clin Psychiatry. 2015;42(4):95-99.

6. Esan O, Esan A. Epidemiology and burden of bipolar disorder in Africa: a systematic review of data from Africa. Soc Psychiatry Psychiatr Epidemiol. 2016;51(1):93-100.

7. Moreno C, Laje G, Blanco C, Jiang H, Schmidt AB, Olfson M. National trends in the outpatient diagnosis and treatment of bipolar disorder in youth. Arch Gen Psychiatry. 2007;64(9):1032-1039.

8. Altshuler LL, Gitlin MJ, Mintz J, Leight KL, Frye MA. Subsyndromal depression is associated with functional impairment in patients with bipolar disorder. J Clin Psychiatry. 2002;63(9):807-811.
9. Soldani F, Sullivan PF, Pedersen NL. Mania in the Swedish Twin Registry: criterion validity and prevalence. Aust NZJ Psychiatry. 2005; 39(4):235-243.

10. Judd LL, Akiskal HS. The prevalence and disability of bipolar spectrum disorders in the US population: re-analysis of the ECA database taking into account subthreshold cases. J Affect Disord. 2003; 73(1-2):123-131.

11. Adegbaju DA, Olagunju AT, Uwakwe R. A comparative analysis of disability in individuals with bipolar affective disorder and schizophrenia in a sub-Saharan African mental health hospital: towards evidenceguided rehabilitation intervention. Soc Psychiatry Psychiatr Epidemiol. 2013;48(9):1405-1415.

12. Onyeama M, Agomoh A, Jombo E. Bipolar disorder in Enugu, South East Nigeria: demographic and diagnostic characteristics of patients. Psychiatr Danub. 2010;22(Suppl 1):S152-S157.

13. Wang W, Ding AA. On assessing the association for bivariate current status data. Biometrika. 2000;87(4):879-893.

14. Fagbamigbe AF, Adebowale AS. A model for measuring association between bivariate censored outcomes. J Mod Math Stat. 2010;4(4): 127-136.

15. Perlis RH, Ostacher MJ, Patel JK, et al. Predictors of recurrence in bipolar disorder: primary outcomes from the systematic treatment enhancement program for bipolar disorder (STEP-BD). Am J Psychiatry. 2006; 163(2):217-224.

16. Christensen KY, Maisonet M, Rubin C, et al. Characterization of the correlation between ages at entry into breast and pubic hair development. Ann Epidemiol. 2010;20(5):405-408.

17. Betensky RA, Finkelstein DM. A non-parametric maximum likelihood estimator for bivariate interval censored data. Stat Med. 1999;18(22): 3089-3100.

18. Gitlin MJ, Swendsen J, Heller TL, Hammen C. Relapse and impairment in bipolar disorder. Am J Psychiatry. 1995;152(11):1635-1640.
Neuropsychiatric Disease and Treatment

\section{Publish your work in this journal}

Neuropsychiatric Disease and Treatment is an international, peerreviewed journal of clinical therapeutics and pharmacology focusing on concise rapid reporting of clinical or pre-clinical studies on a range of neuropsychiatric and neurological disorders. This journal is indexed on PubMed Central, the 'PsycINFO' database and CAS,

\section{Dovepress}

and is the official journal of The International Neuropsychiatric Association (INA). The manuscript management system is completely online and includes a very quick and fair peer-review system, which is all easy to use. Visit http://www.dovepress.com/testimonials.php to read real quotes from published authors. 